\title{
Une alimentation saine et soutenable est-elle possible? Les dilemmes des mangeurs écologistes
}

Leslie Carnoye et Clémence Guimont

\section{(2) OpenEdition}

Journals

Édition électronique

URL : http://journals.openedition.org/developpementdurable/14625

DOI : 10.4000/developpementdurable.14625

ISSN : 1772-9971

Éditeur

Association DD\&T

Référence électronique

Leslie Carnoye et Clémence Guimont, « Une alimentation saine et soutenable est-elle possible ? Les dilemmes des mangeurs écologistes », Développement durable et territoires [En ligne], Vol. 10, $n^{\circ} 2$ | Juillet 2019, mis en ligne le 15 juillet 2019, consulté le 23 septembre 2020. URL : http:// journals.openedition.org/developpementdurable/14625; DOl : https://doi.org/10.4000/ developpementdurable.14625

Ce document a été généré automatiquement le 23 septembre 2020.

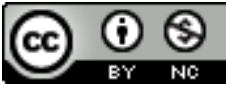

Développement Durable et Territoires est mis à disposition selon les termes de la licence Creative Commons Attribution - Pas d'Utilisation Commerciale 4.0 International. 


\title{
Une alimentation saine et soutenable est-elle possible? Les dilemmes des mangeurs écologistes
}

\author{
Leslie Carnoye et Clémence Guimont
}

1 Manger est un acte tellement essentiel à la reproduction de nos forces vitales qu'il est d'une banalité déconcertante. Et pourtant... l'affaire se révèle de plus en plus complexe. C'est à partir de ce constat qu'a émergé le présent éditorial, non sans liens pour Leslie Carnoye avec sa récente prise de poste dans une école d'ingénieurs en agronomie et agroalimentaire et pour Clémence Guimont avec sa participation au salon des outils pédagogiques de l'alimentation durable organisé par le Douaisis Agglo le 30 avril 2019.

2 Échanger avec les professionnels et les praticiens de l'alimentation (agriculteurs, industriels, collectivités territoriales, associations, chercheurs, mangeurs...) permet de comprendre que nos choix et nos habitudes alimentaires ne relèvent pas de la simple évidence : l'alimentation «fait système ». L'expression "système alimentaire » désigne ainsi « la manière dont les hommes s'organisent, dans l'espace et dans le temps, pour obtenir et consommer leur nourriture » (Malassis, 1994, in. Rastoin et Ghersi, 2010, p. 3). Autrement dit, l'alimentation constitue un enjeu fondamental pour l'ensemble des sociétés humaines et elle n'échappe pas aux rapports de pouvoir propres aux systèmes socioéconomiques et politiques dans lesquelles elle s'insère. Aujourd'hui, le défi est de parvenir à réorganiser un système alimentaire fortement industrialisé, concentré et financiarisé afin de le rendre plus soutenable, d'un point de vue social et écologique (Rastoin et Ghersi, 2010).

3 C'est ainsi que la nécessité de promouvoir une «alimentation durable " (Esnouf et al., 2011) s'est progressivement imposée à partir d'une série de constats. La prévisible augmentation démographique fait réapparaitre la question de la sécurité alimentaire, dans un contexte où les inégalités alimentaires repartent à la hausse depuis les années 1990 à l'échelle mondiale. Par ailleurs, plus de la moitié des calories produites ne sont finalement pas consommées et les problèmes de santé, notamment liés à l'obésité et à une alimentation trop sucrée, salée et transformée, deviennent de plus en plus 
importants dans les pays du nord. En outre, l'agriculture est fortement dépendante des évolutions climatiques, de la disponibilité en eau et de la biodiversité alors que ses impacts environnementaux dans ces trois domaines sont considérables, sans même parler de la tension sur les ressources fossiles qui a déjà et aura sans doute encore plus à l'avenir un impact déterminant sur l'agriculture, l'usage des sols et donc l'alimentation. La crise économique, financière et alimentaire de 2008 a révélé les limites et la dangerosité de la globalisation du commerce des produits agricoles, tandis que la récurrence des controverses, scandales et autres crises sanitaires montre que les problèmes dits "de traçabilité" ne sont pas uniquement la conséquence de déficits informationnels, mais qu'ils sont au contraire consubstantiels à l'existence de filières longues où interviennent de nombreux intermédiaires entre les producteurs et les consommateurs. C'est ainsi progressivement la croyance selon laquelle il suffirait de produire plus pour manger mieux - laquelle est au fondement du système agrialimentaire industriel - qui se trouve ébranlée.

Par contraste, "les régimes alimentaires durables contribuent à protéger et à respecter la biodiversité et les écosystèmes, sont culturellement acceptables, économiquement équitables et accessibles, abordables, nutritionnellement sûrs et sains, et permettent d'optimiser les ressources naturelles et humaines» (FAO, 2010). Entre les deux, dans ce clair-obscur et avec les monstres, se pose donc la question de la transition... et elle se pose avec une acuité particulière lorsque l'appétit nous tiraille et que nous avons la chance de nous retrouver autour d'une table devant notre assiette, voire en cuisine, ou dès lors que notre garde-manger crie famine. Ainsi, la particularité du mangeur écologiste est de se retrouver confronté (au mieux plusieurs fois par jour) à une série de choix parfois cornéliens et à l'issue pour le moins incertaine: au-delà du goût ou de l'envie du moment, que faut-il privilégier et qui faut-il écouter? Les habitudes d'antan? Les nutritionnistes? Les restaurateurs ou les chefs cuisiniers? Les petits commerçants? Les maraichers ou les producteurs? Les étiquettes et les emballages? Le bio ? Le local ? Le commerce équitable? Les AOC ou les IGP ? Les labels rouges? Les nutri-scores ou les applications pour smartphone? Les végétariens, les végans ou les flexitariens? Ou bien encore, tout ce qui ne nécessite pas d'aller au supermarché et de prendre sa voiture ? Et d'ailleurs, quelle part de notre revenu peut-on ou accepte-on de consacrer à notre alimentation? Nous souhaitons ici soumettre à la sagacité du lecteur quelques-uns de ces épineux dilemmes, lesquels restent encore assez largement à résoudre en ce qui nous concerne...

5 Une grande préoccupation du mangeur écologiste peut être de tenter de respecter les recommandations nutritionnelles tout en essayant de réduire le bilan carbone de son alimentation. L'idée, autrement dit, est d'essayer de combiner alimentation «saine » et «durable». En apparence, l'affaire est simple et entendue : il faut commencer par manger moins de viande (et notamment de viande rouge et de charcuteries) laquelle a un impact carbone 3 à 4 fois plus important, en moyenne, que les fruits et légumes (Darmon et Soler, 2011). Cependant, au-delà des chiffres et des réalités moyennes, toute la question est de savoir quelle viande et quels fruits et légumes. Parle-t-on des fruits exotiques importés par bateau ou par avion ou des légumes dits «bio » mais chauffés sous serre, ou bien encore de ceux qui sont issus de d'une agriculture dite conventionnelle, mais locale? Et puis une fois vendus, par quels modes de transports sont-ils acheminés en cuisine? Enfin, comment sont-ils conservés puis cuisinés ? Tous ces paramètres influencent l'impact carbone de notre alimentation. 
6 Il en est donc de même pour la viande. Même si, a priori, la viande blanche présente de meilleures performances carbone ou énergétiques que la viande rouge, rappelons que les ruminants issus de véritables élevages et non pas de productions animales industrielles (Porcher, 2011) participent au maintien des zones humides (lesquelles absorbent aussi du carbone), à la protection des aires d'alimentation de captage en eau potable et au maintien des paysages et de la biodiversité. Ainsi, notre mangeur écologiste se retrouve-t-il tiraillé entre le cycle du carbone... et celui de l'azote, même s'il aura globalement tendance à privilégier (certes, selon son budget et ses capacités culinaires) des produits issus d'une agriculture paysanne et peu transformés plutôt que des produits issus de filières longues et industrialisées (... même si ces dernières peuvent aussi permettre des économies d'échelles intéressantes en termes de carbone).

Plus largement, concernant la controverse sur la question de la consommation de produits d'origine animale ${ }^{1}$, nous avons tendance à penser pour notre part qu'il ne peut être " écologiste » de remettre en cause l'élevage et la relation anthropologique de domestication des animaux, laquelle a historiquement largement participé au maintien de la biodiversité (ce qui est également le cas des semences (Bové et Luneau, 2015)). Pour autant, son pendant industriel et capitaliste est une aberration qu'il est essentiel de parvenir à faire disparaitre ${ }^{2}$. Il s'avère ainsi essentiel, aussi bien d'un point de vue agronomique, à l'échelle des exploitations, que pour le maintien de la diversité des paysages et des dynamiques rurales, de maintenir l'élevage, alors que certains éleveurs se trouvent aujourd'hui dans des situations économiques et administratives très précaires et sont confrontés à de vives critiques ${ }^{3}$. Par conséquent, il nous semble tout à fait possible (et écologique) de réduire notre consommation globale de viande, pour aller vers des produits plus qualitatifs et mieux valorisés. Sous condition d'une déintensification et d'une désindustrialisation des pratiques (y compris d'abattage et de distribution) il parait envisageable de maintenir, voire de développer, dans le même temps, l'élevage.

8 C'est sur la question du poisson que notre mangeur écologiste aura sans doute (encore) plus de mal à suivre les standards d'une alimentation saine. Même s'il faut saluer les efforts faits en faveur de la pêche durable, ou encore l'interdiction de la pêche électrique par l'Union Européenne, manger du poisson plusieurs fois par semaine semble difficilement compatible avec les exigences du développement durable, lesquelles reposent sur un double critère d'égalité intra et intergénérationnelle. C'est donc qu'il serait au contraire "écologiste » d'accepter l'idée de limites au bien-être individuel et de renoncer à une petite partie de ce qui serait optimal en termes de santé pour chacun, afin de pouvoir vivre nombreux et pour longtemps sur terre ? La question est abyssale. Mais nous craignons qu'en l'état, la réponse soit positive.

Une chose établie est que pour maintenir notre équilibre alimentaire, ce n'est pas par n'importe quels fruits, légumes ou céréales qu'il est possible de remplacer une partie de notre consommation de viande ou de poisson, mais par ceux qui sont source de protéines végétales ${ }^{4}$. Or, sur ce point aussi, les associations entre santé et environnement peuvent être plus ou moins heureuses. C'est en effet un grand soulagement, pour le mangeur écologiste, que l'insertion de légumineuses dans les rotations, lesquelles fixent l'azote dans le sol, permettent ainsi de réduire les intrants. Il s'agit de l'un des éléments sur lesquels peuvent s'appuyer les agriculteurs engagés dans un processus de transition agroécologique pour diversifier leurs cultures, quand bien même il reste encore aujourd'hui souvent nécessaire (à la fois pour les 
légumineuses destinées à l'alimentation animale et celles qui sont destinées à l'alimentation humaine) de créer des filières et de se préoccuper de la question des débouchés, de celle de la valorisation et de la répartition de la valeur ajoutée entre les acteurs (Magrini et al., 2017; Meynard et Messéan, 2014). En revanche, le mangeur écologiste est en droit de considérer avec beaucoup plus de scepticisme la production industrielle de spiruline ou encore le soja transgénique et ses dérivés, bien que ces derniers soient plus concentrés en protéines végétales que les lentilles ou les pois chiches bio et locaux...

Un résultat particulièrement intéressant peut être ici mentionné. Darmon et Soler (2011) se sont penchés sur la question de la compatibilité entre une alimentation saine et une alimentation durable et ils ont montré que "la relation entre l'adéquation nutritionnelle des régimes individuels et leur impact carbone est faible, en partie car les régimes nutritionnellement adéquats contiennent des grandes quantités d'aliments à faible impact carbone » (p. 77). Autrement dit, les régimes végétariens ou végans, « toutes choses égales par ailleurs", c'est-à-dire à kilocalories égales et à "équilibre énergétique égal " n'afficheraient pas un impact carbone significativement moins important que les régimes omnivores. Une conclusion majeure de l'étude est en effet qu'une alimentation «durable » est en définitive une alimentation « sobre »: " pour diminuer l'impact carbone de l'alimentation, une voie majeure serait une réduction de la consommation de calories ingérées, indépendamment des familles d'aliments concernés " (ibid.). Ce que montrent par ailleurs Colona et al. (2011) est que la résilience de notre système alimentaire et son écologisation dépendent de la coexistence d'une diversité de sous-systèmes et de conventions de qualité (circuits courts, agriculture biologique, commerce équitable, indications géographiques...) sur lesquels peuvent globalement s'appuyer les mangeurs et les écologistes, même si ces derniers ne sont pas exempts de tensions et de contradictions.

11 En définitive, loin d'être un acte solitaire, manger est une pratique sociale et collective à portée fortement symbolique (Flandrin et Montanari, 1996). Ceci peut expliquer les tensions liées à la coexistence de différents régimes alimentaires au sein d'une société, quand bien même nos contraintes extra-alimentaires (professionnelles, familiales, loisirs, etc.) peuvent aussi s'imposer face aux tentatives d'alignement de nos pratiques alimentaires avec nos convictions politiques et/ou écologistes. Mais c'est aussi la raison pour laquelle ces questions ne peuvent être résolues à l'échelle individuelle. Elles doivent au contraire être débattues, discutées et mises en critique dans les foyers, à l'école, dans les cantines, les restaurants, les champs, les (super)marchés, les collectivités, les entreprises, les associations et les espaces politiques, afin de forcer le capitalisme à mieux traiter notre alimentation. La question est d'autant plus importante que nous ne sommes pas tous à égalité face aux défis de la transition alimentaire, que l'on considère la pyramide des âges, celle des revenus ou celle du capital social.

Pour l'heure, nous espérons que cet éditorial y aura modestement contribué et qu'il aura également permis de mettre ses lecteurs en appétit... pour la lecture notamment du dossier thématique : "Le paysage comme instrument de gouvernance territoriale » coordonné par Marie-José Fortin, Anne Sgard et Magalie Franchomme, sans oublier le reste de notre numéro. 


\section{BIBLIOGRAPHIE}

Bové J., Luneau G., 2015, L'alimentation en otage. Quand les multinationales contrôlent nos assiettes, Autrement, Paris.

Colona P., Fournier S., Touzard J.-M., 2011, « Systèmes alimentaires », Esnouf C., Russel M., Bricas N., 2011, Pour une alimentation durable. Réflexion stratégique duALIne, Quæ, Versailles.

Darmon N., Soler L.-G., 2011, « Impact carbone et qualité nutritionnelle des régimes alimentaires en France », in. Esnouf C., Russel M., Bricas N., 2011, Pour une alimentation durable. Réflexion stratégique duALIne, Quæ, Versailles.

Esnouf C., Russel M., Bricas N., 2011, Pour une alimentation durable. Réflexion stratégique duALIne, Quæ, Versailles.

FAO, 2010, «Definition of sustainable diets ». International scientific symposium Biodiversity and sustainable diets United against hunger, Rome.

Flandrin J.-L., Montanari M. (coord.), 1996, Histoire de l'alimentation, Fayard, Paris.

Magrini M., Anton M., Cholez C., Duc G., Hellou G., Jeuffroy M., Walrand S., 2017, « Transition vers des systèmes agricole et agroalimentaire durables : quelle place et qualification pour les légumineuses à graines ? », Revue Française de Socio-Économie, n 18, p. 53-75.

Meynard J.-M., Messéan A., 2014, La diversification des cultures. Lever les obstacles agronomiques et économiques, Quæ, Versailles.

Malassis L., 1994, Nourrir les hommes, Flammarion, Paris.

Porcher J., 2011, Vivre avec les animaux, La Découverte, Paris.

Rastoin J.-L., Ghersi G., 2010, Le système alimentaire mondial. Concepts et méthodes, analyses et dynamiques, Quæ, Versailles.

\section{NOTES}

1. Dont quelques exemples peuvent être trouvés ici: https://reporterre.net/Manger-de-laviande-sans-culpabiliser-si-elle-vient-de-l-agriculture-paysanne et ici : https://reporterre.net/ Le-veganisme-merite-mieux-que-l-insulte. Sites consultés le 17 juin 2019.

2. Mais dans ce cas, ne vaut-il pas mieux s'en prendre d'abord aux grandes enseignes du fast-food plutôt qu'aux artisans professionnels de la viande ou aux éleveurs?

3. Les lecteurs pourront sur ce point se référer au numéro 231 de la revue POUR, du Groupe Ruralités, Éducation et Politiques de 2016 intitulé « Éloge de l'élevage... Mais quel élevage ?».

4. On considère généralement qu'une alimentation équilibrée contient environ de 10 à $15 \%$ de protéines, de 30 à $35 \%$ de lipides, de 50 à $55 \%$ de glucides et que les apports caloriques journaliers varient en fonction $\mathrm{du}$ sexe et du niveau d'activité physique, d'environ 1800 kilocalories par jour pour une femme sédentaire à 3500 kilocalories par jour pour un homme ayant une intense activité physique. 


\section{AUTEURS}

\section{LESLIE CARNOYE}

Leslie Carnoye est maitre de conférences

Agrosup Dijon, Cesaer (UMR 1041)

\section{CLÉMENCE GUIMONT}

Clémence Guimont est ATER à l'IAE de Nancy et doctorante à l'université de Lille Ceraps (UMR 8026) 